\title{
High-Efficiency Multihomed Multimedia Transmission in Wireless Sensors
}

\author{
Haitao Wang, Hunan International Economics University, China* \\ Yanli Chen, Hunan University of International Economics, China
}

\begin{abstract}
For real-time video transmission in multimedia sensor networks, efficient and reliable communication is very important. However, under actual conditions, when the sensor transmits data to the sink node, the link between the sensor and the sink node usually becomes unstable due to bandwidth limitations and unstable transmission links. Unstable data transmission will affect the normal data transmission of sensor nodes and even cause sensor nodes to fail to communicate with sink nodes. These problems make users who use multimedia sensor networks have a poor user experience. In order to solve these problems, a brand-new, multi-homed, multi-connection system was designed for wireless multimedia sensors, and a prototype system was implemented. Compared with the traditional single-homed connection mode, the system can maintain a more stable connection under the same bandwidth, memory, and CPU resource constraints.
\end{abstract}

\section{KEYWORDS}

Multi-Connection, Multi-Homer, Reliability, Wireless Multimedia Sensor

\section{INTRODUCTION}

Big data represents one of the most profound and most pervasive evolutions in the digital world (Arena, F., \& Pau, G. 2020). Examples of big data come from Internet of Things (IoT) devices, as well as smart cars, but also the use of social networks, industries, and so on. The sources of data are numerous and continuously increasing, and, therefore, what characterizes big data is not only the volume but also the complexity due to the heterogeneity of information that can be obtained. The fastest growth in spending on big data technologies is happening within banking, healthcare, insurance, securities and investment services, and telecommunications. In fact, the definition of big data analysis refers to the process that encompasses the gathering and analysis of big data to obtain useful information for the business.

In recent years, wireless multimedia sensors have developed rapidly, their functions are becoming more and more powerful, and their costs are declining. The development of battery technology has increased the lifespan of these devices and also promoted the development of these devices (SarisarayBoluk P \& Akkaya K, 2015). A solution is introduced based on fuzzy logic aimed at optimizing 
the energy management of electric bikes (Giliberto, M., Arena, F., \& Pau, G. 2019). The design of three-port converters (TPCs) is presented for smooth transitions (i.e., fast settling time, and no obvious overshoot/undershoot) of 7 distinctive operating modes, depending on sources and loads scheduling (Aljarajreh, H.,et al. 2021). A systematic extension of second-order compensated inductive power transfer (IPT) converters is presented (Liu, Y. C., et al. 2021), it is designed for achieving load-independent current (LIC) or load-independent voltage (LIV) output. The rapid development of wireless multimedia sensors has made them widely used in many fields, and the threshold for using these sensors has become lower and lower. Using sensors, cloud servers and clients provided by sensor manufacturers can quickly create a monitoring system.

The widespread use of wireless sensors has brought a lot of multimedia data, such as video sensor data. This type of multimedia data is generally real-time and continuous. Therefore, it is very important to maintain the efficiency and stability of data transmission. In the process of multimedia data transmission, the loss of some data may affect the availability of other data. Due to bandwidth limitations and network stability, the sensor may not be able to obtain a stable connection link when communicating with the server. The instability of the wireless link itself will cause the sensor node to temporarily fail to access the server. A problem with a network node in the link will cause a link to be unavailable. As a result, the connection is abnormally disconnected, the delay increases and the user experience decreases.

Some researchers have conducted research on the reliability of wireless sensors and the reliability of data transmission on wireless sensors, and have obtained some results. The routing protocol optimization in the wireless multimedia sensor network (WMSN) is a method that can control the data flow (Nguyen X T, et al., 2015). This method is very effective in WMSN, but it is not designed for portable smart devices, and it cannot avoid network abnormalities caused by network status. One way to enhance transmission stability in wireless sensor networks is to restore the connection as soon as possible when the connection is disconnected (Joshi Y K \& Younis M, 2014). However, this method will still cause data loss and congestion. Another method is to use multiple connections to reduce delay and enhance the stability of data transmission (Kim D \& Chung K, 2014). This method does not solve the problem of disconnection during data transmission. Finding the shortest path between the sensor and the server to reduce latency is also a way to enhance stability. In distributed and independent sensors, this method is effective, but when the network condition is not good, the delay will still occur, and this method mainly focuses on improving energy utilization (Chaudhary D D \& Waghmare L M, 2013). Multi-homed (multi-homed) is also an available mode to improve data transfer. Multi-homing means that a device has multiple addresses for other devices to access, thereby enhancing the stability of data transmission (Sousa B M, et al., 2011; Wallace T D \& Shami A,2012; Capela N \& Sargento S, 2014; Kuntz R, et al., 2013). However, it is unrealistic for each device to hold multiple addresses, and it is also very difficult for all devices to support multi-homed protocols.

There have been several studies dedicated to improving the reliability of wireless sensor networks, and some studies have even improved the reliability by adjusting routing protocols. A wireless sensor system is proposed for measuring mechanical operation, the integrity and correctness of data transmission are ensured (Lee H C, et al., 2014). A novel congestion avoidance protocol is proposed based on decentralized rate control, which is an adaptive rate control algorithm based on a combination of discrete proportional integral differentiation (PID) control method and single neuron (Li G H \& Li J Z, 2011).

Many researchers have also conducted research on multi-connection, and some multi-connection mechanisms are proposed to enhance multimedia services. TCP-FIT is a brand new TCP congestion control algorithm, which performs well under a wide range of network conditions (Wang J Y, et al., 2011). TCP-ROME is a multi-TCP connection protocol that enables real-time multimedia services to overcome network bottlenecks and bandwidth fluctuations (Park J W, et al., 2011). An adaptive TCP multi-connection mechanism is proposed to improve the quality of video in wireless networks (Kim 
D \& Chung K, 2014), it can maximize the quality of service while minimizing the total distortion of the video stream.

With the continuous development of Internet technology, people's demand for Internet applications has gradually increased. At the same time, with the advancement of communication technology, the diversification of access technologies and the reduction of access equipment costs have enabled users to use multiple communication paths for business operations. Parallel transmission can increase the service throughput rate and meet the transmission service requirements of both parties in a shorter time. In some systems with strict performance requirements, redundant backup schemes are always used at various levels to prevent accidental data loss. When applied to communications, it evolves into a multihomed mechanism. However, with the rapid development of home networks, vehicle networks, and office networks, multi-homed implementation mechanisms can also be used in personal area network communications to provide redundant data backup and improve the reliability of data transmission. At the same time, multi-stream and multi-path transmission can be used to improve the throughput of the communication link. multi-homing is also a hot area for researchers (Al-Najjar, A., Khan, F. H., Portmann, M., 2020; Ma, P. F., You, J. L., Wang, J. L., 2020). The multi-homing transmission of high-definition video with high transmission delay requirements is mainly investigated in wireless networks (Wu J Y, et al., 2016). In order to solve the problems encountered during transmission, a new delay stringent coded transmission framework is designed. An adaptive network interface selection mechanism is designed based on Q-Learning to ensure QoE (quality of experience) for multi-hosted devices in the Internet of Things (Souihi S, et al., 2016). A loss tolerant bandwidth aggregation (LTBA) mechanism is designed for multi-homed devices in a wireless environment (Wu J Y, et al., 2014). This mechanism can reduce continuous packet loss in the case of sudden loss.

With the increase of the usage of multimedia applications, the next generation of wireless networks need to satisfy the quality of the transmitted application even if the network is characterized by limited radio resources such as bandwidth (that it is not less than that required by all the traffic sessions). Many call admission control (CAC) and bandwidth degradation algorithms for real time transmissions were proposed. A algorithm is proposed to minimize the risk of delay violation by facilitating the triggering of the CAC algorithms (Chaari H,et al., 2020). The objective is to adapt the available bandwidth of the wireless resources to be ready to admit any new session without degrading the QoS of already existing sessions. A bandwidth level degradation algorithm is presented to reduce the bandwidth of some active sessions in the cell in order to accommodate the arrived calls. However, CAC algorithms may consume intensive processing time that can provoke delay constraint violation for new calls and handover calls, in particular when it deals with real-time transmission. All this could cause the degradation of the quality of service (QoS) requirements. Energy-efficient and robust face detection and recognition scheme can be useful for many application fields such as security and surveillance in multimedia and visual sensor network (VSN). VSN consists of wireless resources-constrained nodes that are equipped with low-energy CMOS cameras for monitoring. On the one hand, captured images are meaningful multimedia-data that impose high energy consumption to be processed and transmitted. On the other hand, visual sensor (VS) is a battery-powered node with limited life-time. This situation leads to a trade-off between detection-accuracy and power-consumption. This tradeoff is considered as the most major challenge for applications using multimedia data in wireless environments such as VSN. For optimizing this trade-off, a novel face detection and recognition scheme has been proposed based on VSN (Zam A, et al., 2020). In this scheme, detection phase is performed at VS and recognition phase is accomplished at the base station (sink).

The computer in the network may have multiple network cards inserted, and each network card represents an interface of the computer. Of course, this computer also has multiple physical addresses, and such a computer is called a multi-host computer. The mode of connecting to the backbone network through only one link is prone to a single point of failure and cause network paralysis. In this paper, a new multi-homed connection mode is designed for wireless multimedia sensors. This multi-homed 
system can ensure the stability and reliability of the sensor in an unstable network environment. A transmission mechanism is designed. The transmission mechanism can be changed in a variety of network environments. In a wireless environment, the device can choose a variety of data transmission methods, which enhances the flexibility of the system.

\section{SYSTEM ARCHITECTURE}

The structure of the multi-homed system is shown in Figure 1. The servers in the system have multiple hosts with different IP addresses. The sensors in the system can establish multiple connections with different hosts at the same time, and transmit data through these connections. For security, the receiver should know the identity of the connection person and can obtain this identity by using sensor information.

\section{Multi-Homed Mechanism}

Figure 2 is the traditional sensor network mechanism, and Figure 3 is the multi-homed sensor network mechanism. The transmission of multimedia data will be affected by the stability of the connection between different networks. The multi-homing mechanism uses multiple networks for transmission, avoiding the transmission being affected by the instability of one network. This mechanism can improve the reliability of sensor nodes and reduce the probability of unstable transmission.

Figure 1. Multi-Host System Architecture

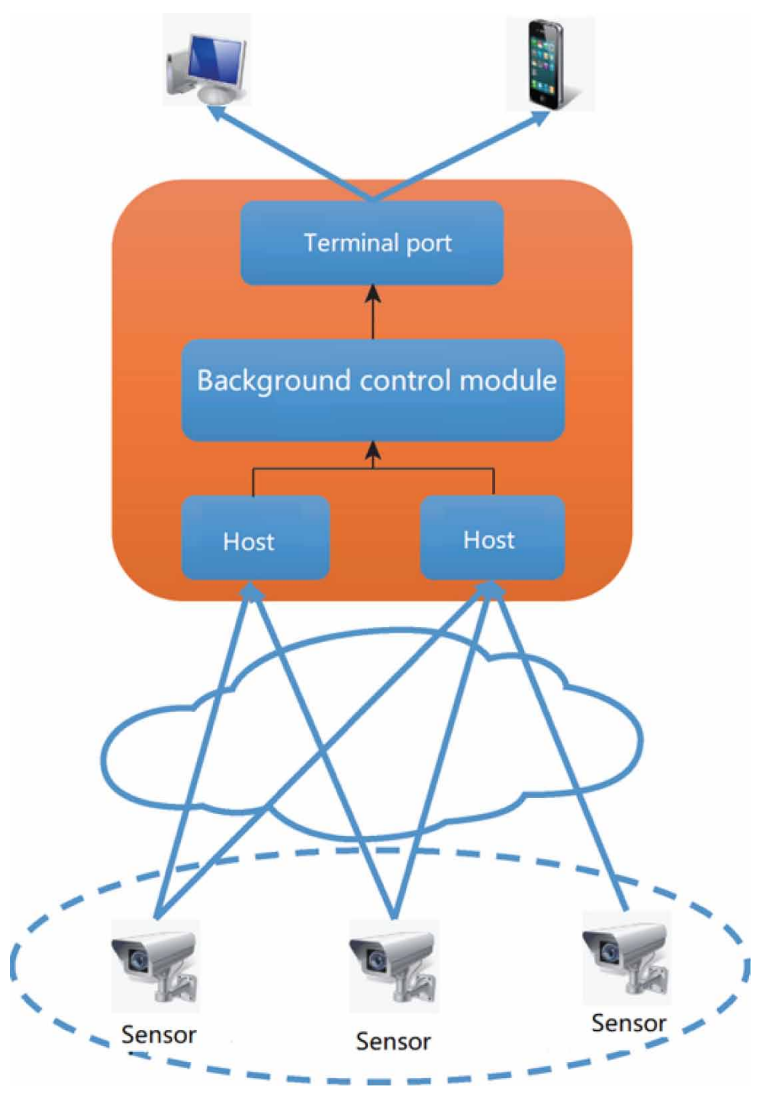




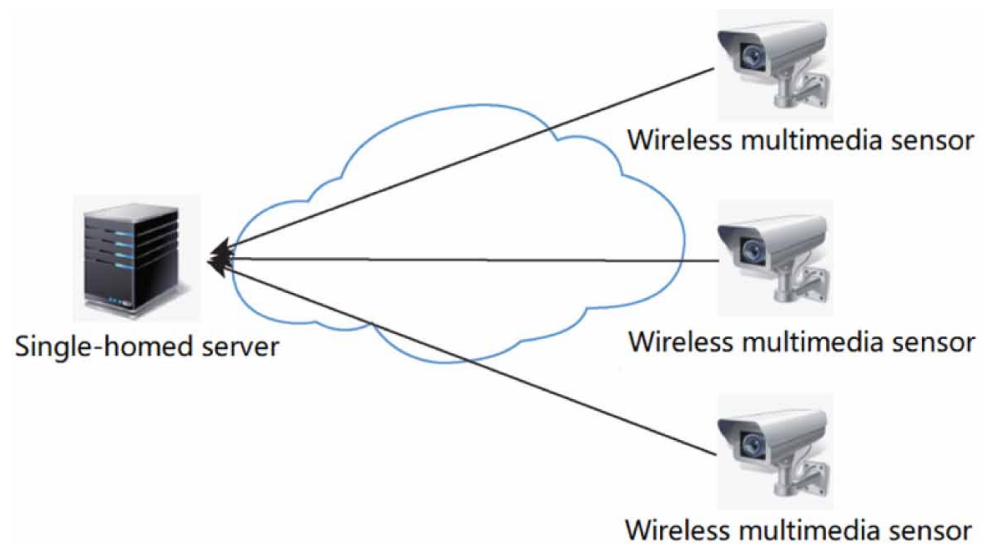

Figure 3. Multi-homed transmission mechanism

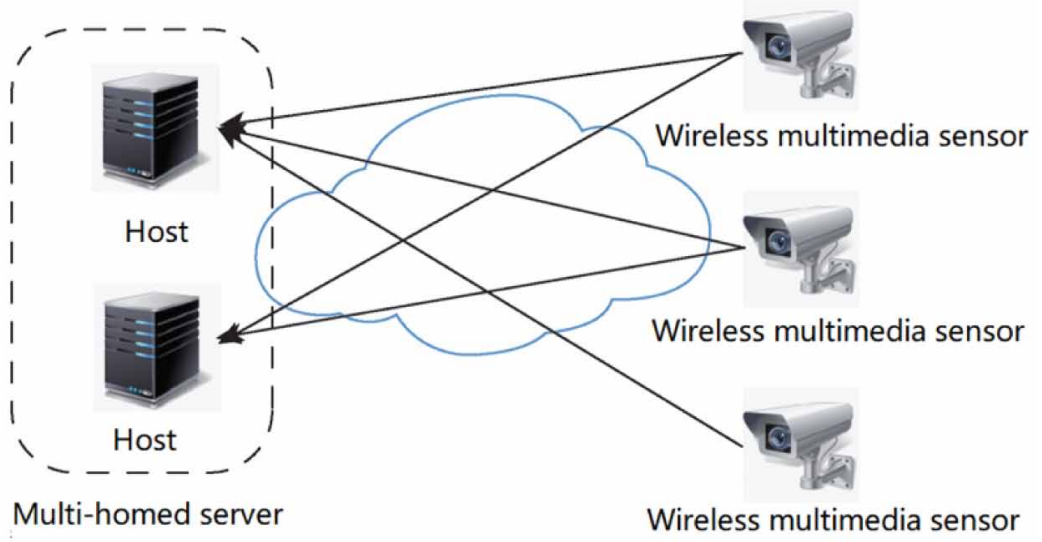

The multi-homing mechanism allows the server to have multiple "hosts" with different addresses. Sensor nodes can access multiple hosts. In sensor nodes, in order to obtain different connection states, the end points of the connection need to be different hosts.

The server of the traditional mechanism has only one access point for the sensor to connect. Under the multi-homing mechanism, when a certain link between the server and the sensor node is unstable, as long as the other links are normal, the sensor can still transmit data.

The multi-homing mechanism is still available for traditional sensors. It is not mandatory to establish multiple connections between the sensor and the server. Traditional sensors can only establish a connection to transmit data. Multi-host sensors can also be integrated into traditional single-host systems in this way.

\section{Multi-Homed Server}

The multi-homed server module is the part that verifies the connection and forwards data, and is also the basis of the multi-homed mechanism. Figure 4 shows the structure of this module. A multi-homed server has several hosts, a terminal port, and a background control module. The sensors connect to different hosts and establish connections to transmit data. The multimedia data is transferred to the 


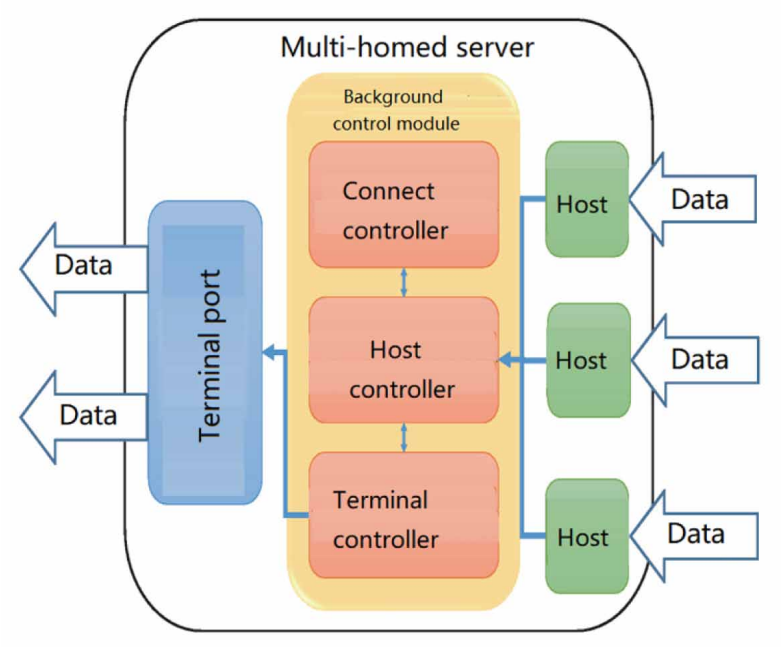

server through the host and processed and identified in the background control module. The terminal accesses the server through the terminal port and requests the server to forward data. Legal data will be forwarded to the terminal through the terminal port.

Each host has an independent IP to receive sensor data. The host can directly establish a connection and control the disconnection. When a host receives a request to establish a new connection, it will pass the request to the background control module and obtain the request verification result.

The terminal port is the portal through which the terminal communicates with the server. This module directly controls the communication with the terminal, and all terminals send requests and receive replies through this port. When a terminal needs to get data from the server, it needs to send the request to the terminal port. At the same time, data is also transmitted to the terminal through this port.

The background control module is a multi-homed server controller. As a controller, this module can control other parts of the multi-homed server. It can not only start and stop other modules, but also process requests from outside and return processing results.

The data enters the system by the host and is processed by the background module, and the terminal port cannot obtain data directly from the host. The host sends the unprocessed data to the background processing module, and the background processing module checks the validity of the data. The legal data will be recorded and sent from the terminal port to the terminal with demand as required.

One advantage of the multi-homed system is that it provides multiple data transmission modes for sensors in different network environments. The connection between the sensor and the host can be many-to-many, and different hosts can simultaneously receive data from the same sensor. Because the connection status between the sensor and different hosts is different, the probability of the sensor being disconnected from all hosts is low. When one connection is disconnected, the sensor can send data to the server through other connections. Multi-connection is a prerequisite for the sensor to improve its reliability.

Another advantage of the system is that when a host actively shuts down or cannot connect, the sensor is still available. When a host is closed, all connections connected to this host will be shut down immediately, and other hosts can work normally. For the sensor, it can abandon this abnormal host and send data to other hosts. Therefore, a sensor can always find the access point to connect to 
the server, reducing the occurrence of dropped connections. This also enhances the reliability of the entire system.

Traditional sensors can only maintain one connection, it can also connect to a host to access the server. In some cases, multi-homed sensors do not need to maintain multiple connections. In a multihomed system, the number of connections maintained by the sensor is variable, and the flexibility of the sensor is higher.

\section{Multi-Homed Sensor}

Multi-homed sensors are wireless devices that can generate and transmit multimedia data with batteries or continuous power supply in a Wi-Fi environment. Figure 5 illustrates the structure of a multi-homed sensor. A multi-homed sensor has a transmission module, a data control module and several data sources.

A multi-homed sensor can have multiple multimedia data sources, so that different types of data can be generated at the same time. A data source can also produce sensor data of different qualities. The function of the data control module is to control these data sources. It can manage all data sources and select the data to be sent. The module cannot send data directly to the server after obtaining the data.

The data transmission module is the core of the multi-homed sensor. This module controls all communications between the sensor and other devices, including the host, and can detect whether a connection is available. When the connection is interrupted, it will send data through other available connections and try to reconnect the current connection. When there is data to be sent, the module will select several of all available connections to transmit the data.

Traditional sensors usually have only one connection. The instability of the network will cause the connection to fail, this kind of problem will cause the interruption of data transmission, and cause the loss and delay of data. Multi-homed sensors solve this problem by maintaining multiple connections. Compared with other multi-connection sensors, the main difference of multi-homed sensors is that multiple hosts with different IPs can be used for data transmission, which makes multi-homed sensors more stable than traditional multi-connection sensors. Although traditional multi-connection sensors have multiple connections, all connections are the same. When the sensor is connected to the host's network, all connections may be affected. Multi-homed sensors can sort the connections based on

Figure 5. Multi-homed sensor

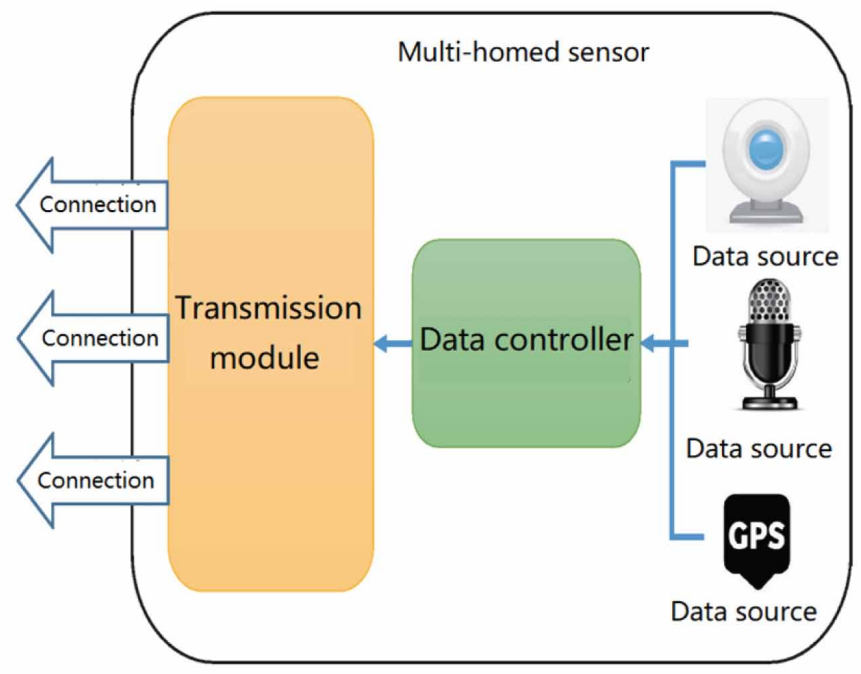


the number of connection failures in history and select the most stable connection. When the most stable option fails, the multi-homed sensor can also switch to a relatively stable connection.

The following features can reduce the delay of sensor data transmission:

1. The multi-homed server allows sensors to select the most suitable host according to their needs, which can reduce the consumption of sensor resources such as bandwidth.

2. One connection regards other connections as a hot backup. When one connection is disconnected, the multi-homed sensor can use other connections and restart the disconnected connection.

3. The probability of simultaneous disconnection is greatly reduced, which will enhance the reliability of the sensor.

\section{Multi-Homed Terminal}

A multi-homed terminal is a device that receives data from a multi-homed system. The terminal can only obtain data from the multi-homed server, but not directly from the sensor. For the sake of safety and flexibility, the terminal needs to know all the connections of the sensor, which means that the terminal can obtain all the connection verification information from the sensor information. When a device only needs part of the sensor data, it can select the specified connection to obtain the information.

\section{Connection Quantity Control}

Not all sensors can install larger memory and stronger processors. For those sensors with more resources, connections can be established with all hosts at one time when establishing connections, and idle connections are used as backups. This connection establishment method is not suitable for sensors with fewer resources, and the amount of data generated by some sensors is not very large, and there is no need to use too many connections for transmission. Too many connections also occupy the host's resources, resulting in the failure to establish some data transmission connections, which reduces the availability and stability of the system.

In order to control the number of sensor connections, multi-homed sensors need to adaptively adjust the number of connections. New connections can be created when the network is unstable, and the number of connections can be reduced when the network is stable.

The main function of redundant connections is to replace failed connections. The number of redundant connections is determined by the number of connections that may fail. When the system starts, the number of connections is determined by the amount of data generated by the sensor, and only one redundant connection is needed at this time. When there is a problem with the data transmission connection, it is necessary to replace the problematic connection with a redundant connection. After the problematic connection is restored, it is converted to a new redundant connection, and a new redundant connection is established at the same time. If there is a problem with a redundant connection, the connection is discarded. It can be seen that the number of redundant connections is equal to the number of problematic connections in the most recent period plus one. This firstly ensures that there is a backup connection for the connection that may have problems again in the recent period; secondly, the problematic connection is placed in the redundant connection, and the connection can be completely deleted when the connection problem occurs again, and the problem-prone connection is gradually deleted from the system. If the connection failure problem does not occur for a long enough period of time, the number of connections can be halved to reduce the resource usage of the system.

\section{Multi-Connection Transmission Protocol}

Through multiple connections, the sensor can select a suitable protocol to transmit data in different environments. This section will introduce the three most commonly used transmission mechanisms. 
For convenience, suppose a sensor has two connections A and B, and they can generate video and audio data.

As shown in Figure 6, the first protocol is that the sensor only uses connection A to transmit data, and connection B is the hot backup of connection A. Unless connection A is unavailable, connection B will not be used. This is the simplest protocol and the easiest to control for sensors. When different data are highly correlated, the sensor can choose this protocol to transmit data. When using this protocol for transmission, the transmission module is relatively simple to control, and the data can arrive in the correct order, but when the delay occurs, all the data generated by the sensor will be affected. When the amount of data is large, the transmission load of a connection will also be relatively large, thereby affecting the effect of transmission.

As shown in Figure 7, the second protocol is that the sensor uses connection A and connection $\mathrm{B}$ to transmit different data respectively. If connection $\mathrm{A}$ is disconnected, connection $\mathrm{B}$ will connect to A's data and wait for A to reconnect, or replace A with a redundant connection. In this protocol, a connection usually only transmits one type of data, and other connections are regarded as its hot backup. When using this protocol, the delay of one connection will not affect the data transmission of other connections. However, this transmission protocol is only an improvement of the first transmission

Figure 6. The first transmission protocol

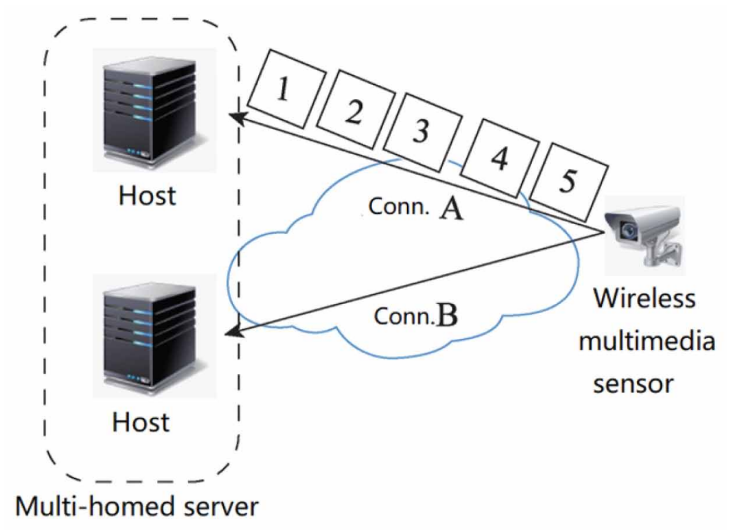

Figure 7. The second transmission protocol

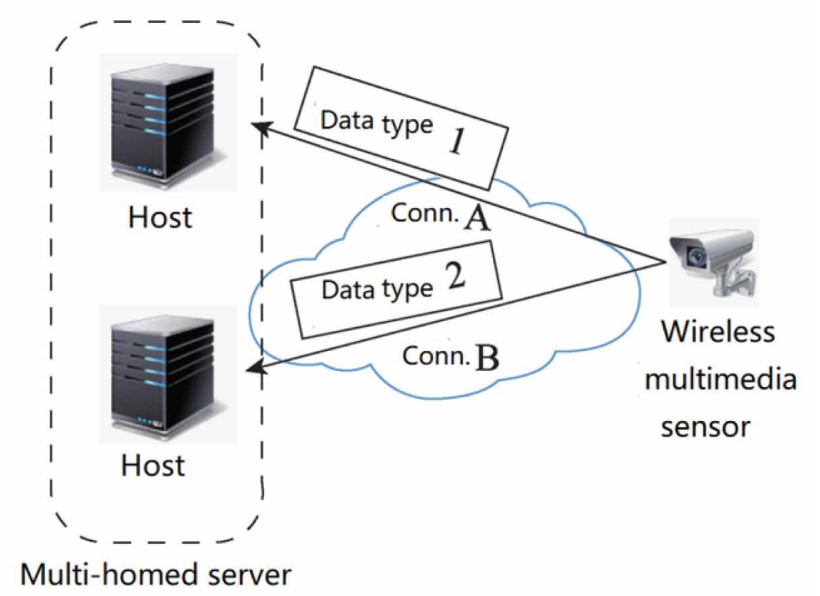


protocol, so the problem of a large connection load will still occur when the amount of data is large. At the same time, if different types of data have a strong connection, this transmission protocol can also cause problems.

As shown in Figure 8, the third protocol is that the sensor uses connection A and connection B to alternately transmit data. The data transmission module of the sensor will pack the data into groups according to the characteristics, and use different connections to transmit the data alternately on a group basis. The data in the same group have a strong correlation. This protocol combines the advantages of multiple connections alternate transmission, if fully reduces delays and improves service quality. However, in this protocol, the sensor is required to add the data number to the data packet before transmitting the data, the data is prevented from being out of order. At the same time, a connection with the same transmission rate and delay should be selected for transmission to reduce the possibility of data arriving in out of order. When the send data is with low latency and the sensor knows how to group the data, this protocol can be selected.

The three protocols can have different effects in different network environments. The network environment in which wireless multimedia sensors work is changeable, and the network environments referred to in the design of the three protocols are different.

- The first protocol can be applied to most network environments. This is because there is actually only one connection for transmission under the first protocol, so the efficiency of multi-connection mode transmission depends on the connection for transmitting data. Under the first protocol, the disconnected loss is can be reduced by increasing the number of connections as a backup. The first protocol does not need to consider the network environment too much when using it, but when the sensor hardware resources are not many, the first protocol will occupy more system resources and cause a certain waste of resources.

- The second protocol requires the same number of connections and data types when actually transmitting. The stability of data transmission mainly depends on the connection that transmits this data. A method like the first protocol is used by increasing the backup connection to reduce the loss when the connection is disconnected. There are two shortcomings, one is the same as the first protocol, the second is that if the two connections are in different network environments and the transmitted data is highly correlated, it may cause the data to be out of sync and cause the required cache to be excessive. More, it will also cause a certain waste of resources.

- The third protocol can improve the connection transmission capacity and reduce the delay during actual transmission, but the problem is also obvious. First, it requires users to design the transmission

Figure 8. The third transmission protocol

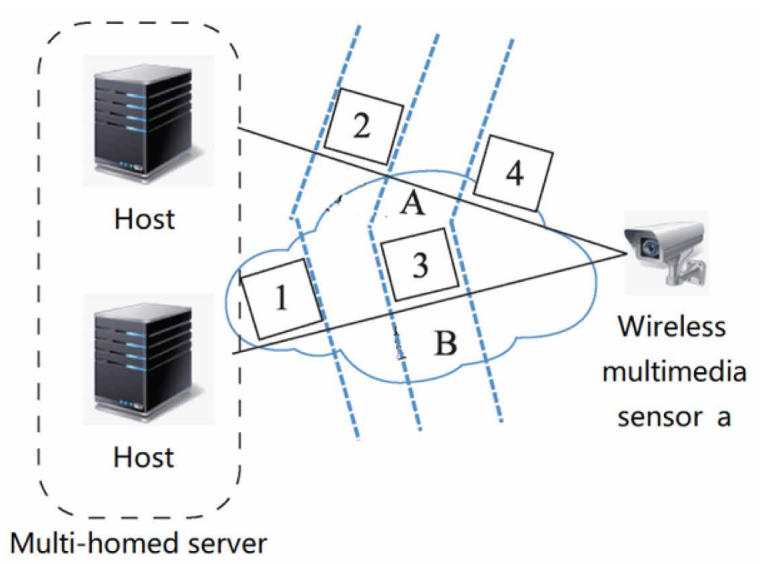


packet mode, which is more complicated than the other two protocols; second, it requires the connection status for transmission to be similar, the asynchrony of data is reduced; third, it requires the system to provide a certain buffer, there are certain requirements in system resources.

\section{Multi-Homed Service Route Selection Method}

After the router matches each destination address (multi-destination address data packet) by forwarding, it obtains multiple forwarding ports, and how to choose among multiple forwarding ports will determine the transmission performance. This article tries a variety of path selection methods. The first is the basic random selection and polling selection methods. Random selection will randomly select one of the ports that can be forwarded, and polling selection will select one by one to achieve port load balance. In addition, the selecting method is used according to the port status, the router has a state flow table that records the port. The table records the state value of the router port, including state information such as bandwidth, load, and delay. The router compares the state value of each forwarding port, a port with a better state value is selected for forwarding, so as to improve the efficiency of forwarding.

\section{PERFORMANCE ANALYSIS}

What is the performance of the multi-homed wireless sensor system and the traditional wireless sensor system? In the system of this article, a sensor test program is implemented on an open source Android development board. The system version of the device is Android4.2, the CPU model is A20 ARM Cortex A7 Dual-core, and the running memory is $1 \mathrm{~GB}$. The test network is a wireless network with a bandwidth of $100 \mathrm{Mb} / \mathrm{s}$. The multi-homed server (Huawei FusionServer Pro 1288H V6) used for testing is two hosts $\mathrm{A}$ and $\mathrm{B}$ with different addresses. The experiment uses bytes per second to show the rate at which the sensor transmits data under stable and unstable network conditions.

\section{Transmission Under Stable Network Conditions}

In this test, 3 multi-homed sensors and 3 traditional sensors are installed in a stable Wi-Fi network. These sensors collect and send the same multimedia data. No other programs in the network occupy the network bandwidth, and the network environment is stable, so all sensors can communicate with the server stably. Two identical clients simultaneously request and receive multimedia data generated by these sensors.

It can be seen from the test results that the multi-homed sensor exhibits the same performance as the traditional sensor. The three protocols defined in the article show the same transmission performance in a stable Wi-Fi network environment. This result proves that multi-homed sensors can achieve the performance of traditional sensors under stable network conditions, and it shows that multi-homed sensors can replace traditional sensors without causing an increase in network bandwidth usage.

\section{Transmission Under Unstable Network Conditions}

In this test, multi-homed sensors and traditional sensors are installed in an unstable Wi-Fi network to transmit these data. The first experiment is the active shutdown of the host. The sensor will close all connections to one of the hosts on the server. The second experiment is to block the designated host. The route from one host of the server to all devices will be interrupted, and all devices cannot communicate with this host. The sensor needs time to detect the anomaly and try to reconnect.

Figure 9 and Figure 10 show the sensor data transmission rate in the first experiment. Figure 9 is the data transmission rate when the connection between the sensor and host A is cut off, and Figure 10 is the data transmission rate when the connection between the sensor and host B is cut off. In the experiment, the sensor immediately found the abnormality and reacted quickly. It can be seen 
Figure 9. Connections between home $\mathrm{A}$ and sensor disconnect forwardly

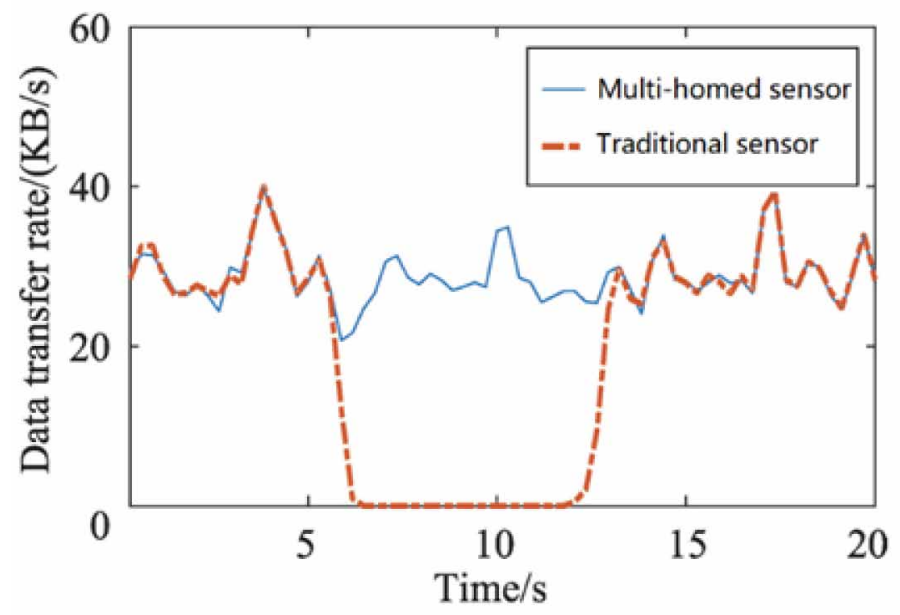

Figure 10. Connections between home B and sensor disconnect forwardly

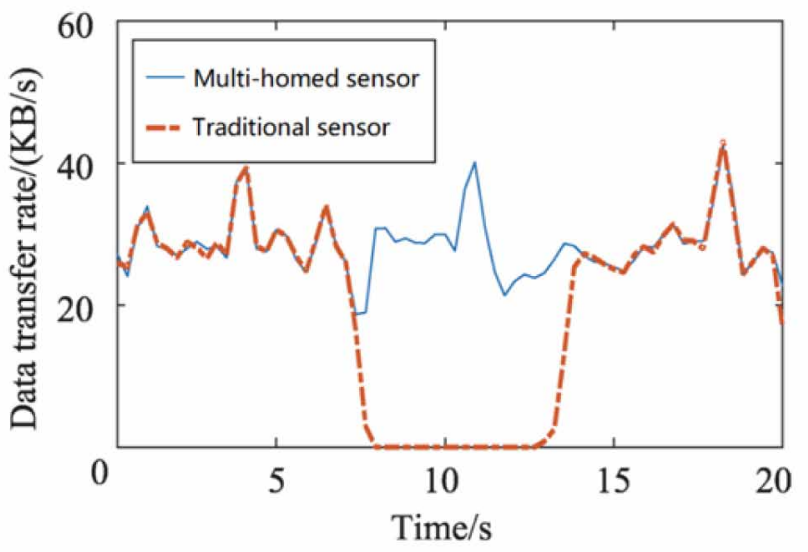

from Figure 9 and Figure 10 that the transmission rate of the traditional sensor quickly drops to zero because its only connection is cut off and disconnected from the server.

The multi-homed sensor can still transmit data normally in the experiment, and the data transmission rate is hardly affected. This means that a multi-homed sensor can transmit data with other hosts when one host is closed, which traditional sensors cannot do. Therefore, multi-homed sensors have higher stability than traditional sensors.

Figure 11 and Figure 12 are the sensor data transmission rate in the second experiment. Figure 11 is the data transmission rate when the route between the sensor and host A is cut off, and Figure 12 is the data transmission rate when the route between the sensor and host B is cut off. It can be seen from Figure 11 and Figure 12 that the traditional sensor was severely affected in the experiment, and the transmission rate was reduced to zero in the experiment. However, the multi-homed sensor is not greatly affected in the experiment. No matter which link is cut, the multi-homed sensor works more stably than traditional sensors, and it can transmit data normally when an attack occurs, reducing 
Figure 11. Connections between home $\mathrm{A}$ and sensor cannot transmit data normally

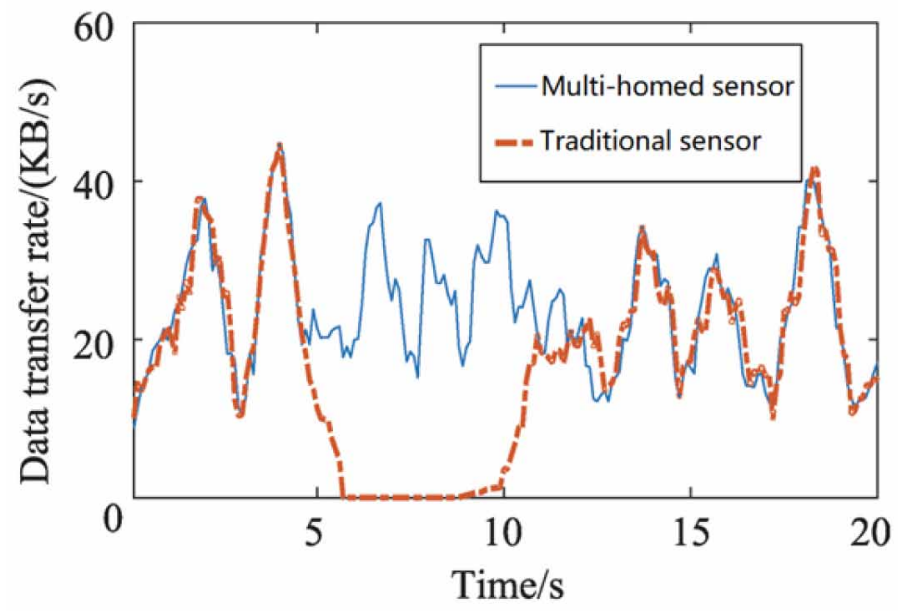

Figure 12. Connections between home B and sensor cannot transmit data normally

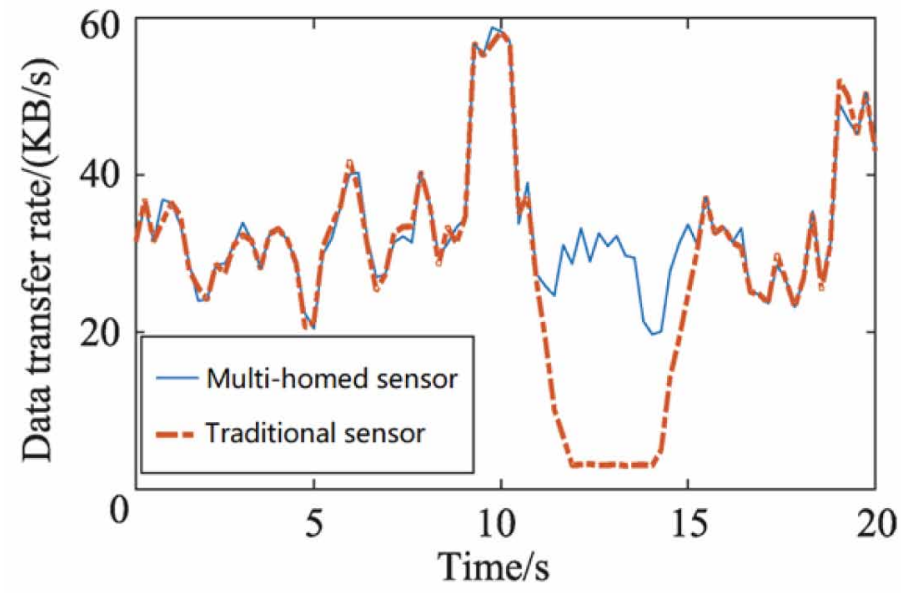

data loss. Under unknown network conditions, a host is likely to be temporarily unavailable due to various reasons. Multi-homed sensors can select other hosts to reduce the probability of disconnection.

It can be seen from the test that the multi-homed sensor is more stable than the traditional sensor. When a connection is actively or passively disconnected, the multi-homed sensor can continue to communicate with the server, reducing the occurrence of dropped connections.

\section{Performance Test}

This article runs multi-homed sensors and traditional sensors and makes them work properly. Figure 13 shows the CPU usage during this time. The CPU occupancy of multi-homed sensors fluctuates at $70 \%$, which is similar to traditional sensors. There is no additional CPU usage for the management of multiple connections. Figure 14 shows the memory usage during this time. The memory usage of multi-homed sensors is still similar to that of traditional sensors, both around $19 \mathrm{MB}$. Figure 13 
Figure 13. CPU usage of sensors

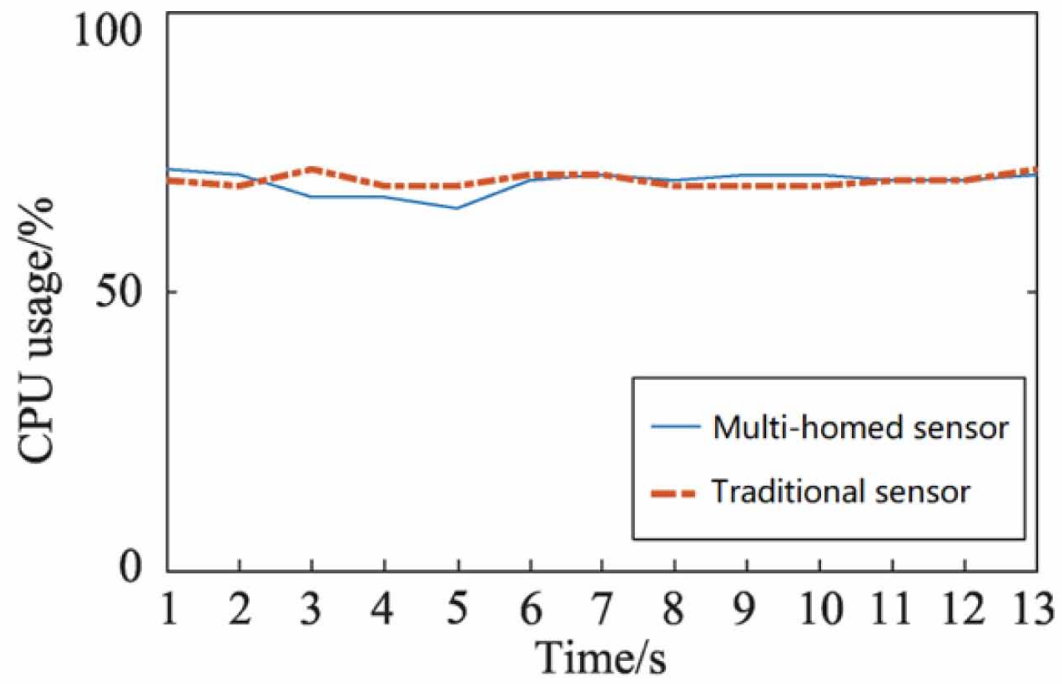

Figure 14. Memory usage of sensors

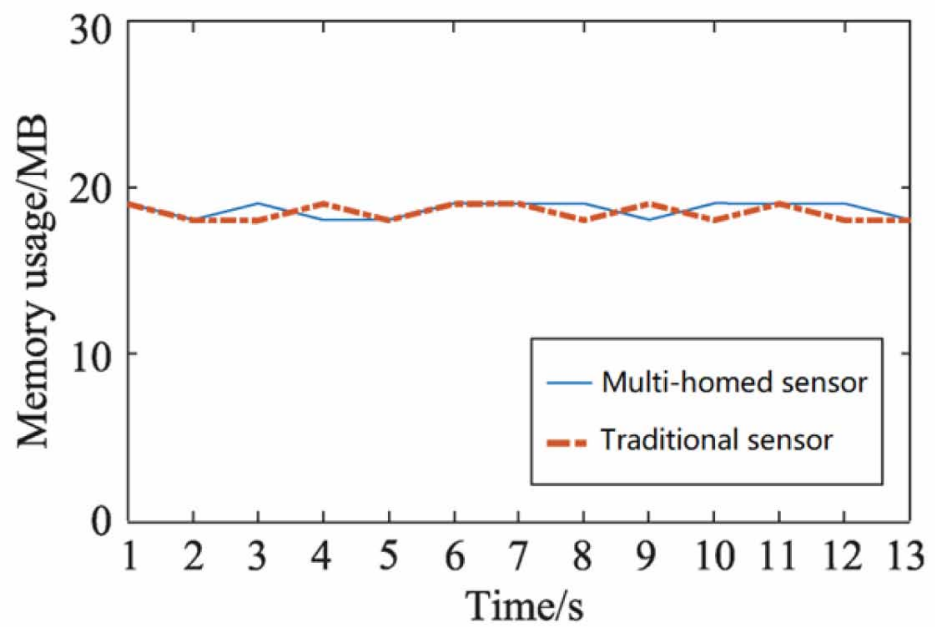

and Figure 14 show that there is no significant difference in the CPU and memory usage of the two sensors. Multi-homed sensors do not need to occupy additional resources than traditional sensors, and it can obtain higher stability. When upgrading a traditional sensor to a multi-homed sensor, there is no need to install a new CPU and memory. This point also applies when using portable smart devices as multimedia sensors.

\section{Energy Consumption Analysis}

In wireless sensor networks, the modules of energy consumed by nodes mainly include sensor modules, data processor modules, and wireless communication modules. From the perspective of wireless sensor network operation, energy consumption operations are mainly divided into two categories: 
communication-related energy consumption and non-communication-related energy consumption. The energy consumption related to communication is mainly concentrated on the energy consumption of the sensor module to collect data and the data processing module to process the data. Normally, the amount of information collected by sensor nodes is not large, and data processing is simple. Therefore, the power consumption of sensor modules and data processing modules is low. Most of the energy consumption of nodes is in the operation of wireless communication modules to realize data transmission between nodes. The energy consumption related to communication mainly includes the data sending of the data source node, the data receiving and forwarding of the intermediate node, and the data receiving of the destination node. Among them, the sending end is responsible for sending local data packets, control packets, and transit data packets sent by other nodes, and the receiving end is responsible for receiving data packets and control packets.

The wireless communication module has four working modes according to the order of power consumption: Transmit, Receive, Idle, and Sleep. In a wireless sensor network, when a node neither sends nor receives data, the wireless communication module is usually in idle mode. It always monitors the usage of the wireless channel and checks whether there is data sent to itself, so its circuit is active. The power consumption is still high. When the node is in a sleep state, the power consumption is very low. Except for sleep mode, the other three are in active state.

The use of efficient communication protocols is the key to reducing the overall energy consumption of wireless sensor networks. 100 nodes are randomly arrange in the $100 \mathrm{~m} \times 100 \mathrm{~m}$ area, from the average energy consumption (in the network survival process, the average energy consumption of each node during data transmission, data perception, data reception, and data fusion) performance, it is analyzed and compared with LEACH (Low-Energy Adaptive Clustering Hierarchy) (Radhika, M., Sivakumar, P., 2021), as shown in Figure 15. The research results show that the method in this paper is more effective than LEACH.

Figure 15. Energy dissipation comparison

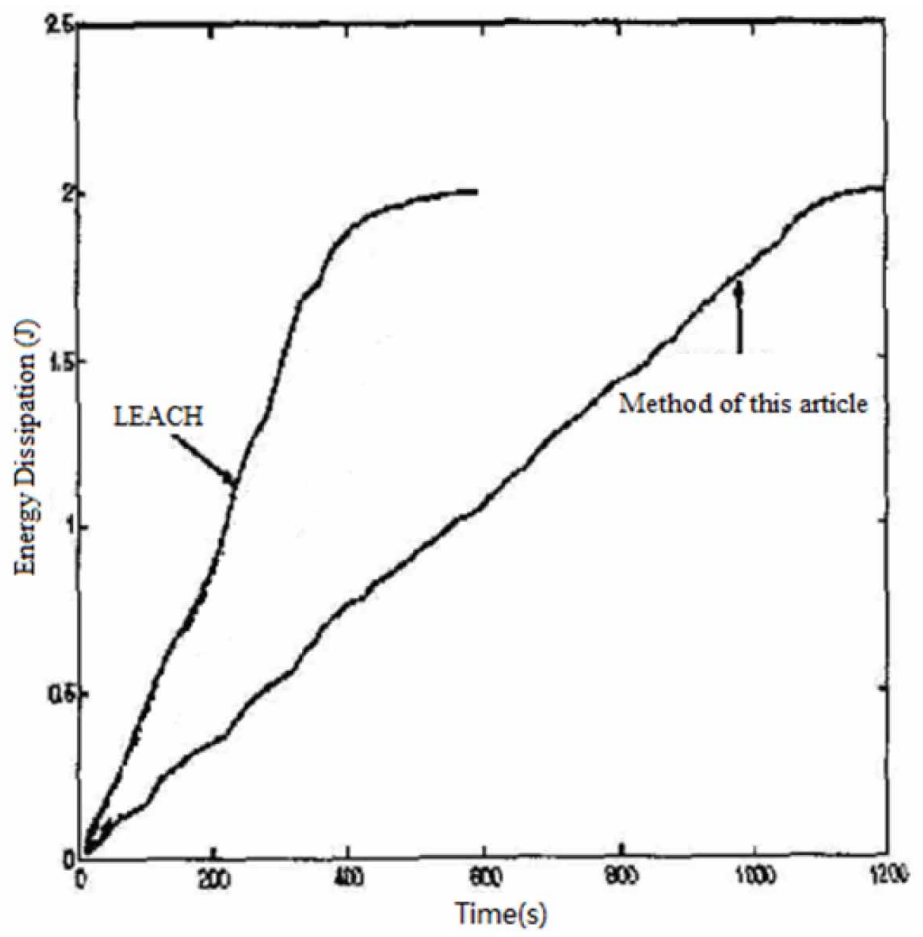




\section{CONCLUSION AND OUTLOOK}

Multimedia technology is a technology that uses computers to store and manage various information such as language, data, audio, and video, so that users can communicate real-time information with the computer through multiple senses. The content displayed and carried by multimedia technology is actually the product of computer technology. Multimedia communication technology is actually a combination of communication technology and multimedia technology. It uses various types of media information to comprehensively process various data, audio and other materials. Multimedia communication technology integrates the advantages of multimedia and communication technology. It has the characteristics of interactivity, compositeness, distribution, authenticity, etc., and it can provide people with a variety of information services.

With the advent of 5G, multi-homing will be an increasingly common scenario, which is expected to increase transmission rates, improve transmission reliability, and reduce costs for users. From the perspective of the network level, the network topology of wireless sensors is changeable, and there is uncertainty in the network topology. For example, the sensor nodes constituting the network topology can be increased or decreased at any time, and the network topology diagram can be separated or merged at any time. Security is not high. The wireless sensor network transmits information wirelessly, so the sensor nodes are easily invaded by the outside world in the process of transmitting information, which leads to the leakage of information and the damage of the wireless sensor network. Most of the nodes of the wireless sensor network are exposed. It greatly reduces the security of the wireless sensor network.

In this paper, a new wireless multimedia sensor system is proposed, it can work stably under unstable network conditions. The structure of the system is designed and implemented. Its advantage is that the sensors in the system are more stable. In order to enable the sensor to run in multi-homing mode, the sensor uses a connection number control mechanism to control the connections held by the sensor. In order to make the system run more flexibly in different environments, three sensor transmission protocols are designed in this paper, and their advantages and disadvantages are analyzed. Finally, three experiments were carried out to test the performance of the system. From the test, it can be found that in the system of this article, the sensor can complete the same data transmission work without increasing resource consumption, and there is higher stability.

In this paper, a new multi-homed connection mode is designed for wireless multimedia sensors. Compared with single-homed wireless multimedia sensor system, this article mainly focuses on the following aspects.

A multi-homed system architecture is designed for wireless multimedia sensors. Under this system architecture, a multimedia sensor can be connected to multiple server hosts at the same time. These host addresses are different, and the sensor can transmit data through multiple different connections. The multi-homed system in this paper can ensure the stability and reliability of sensors in unstable network environments, and the problems caused by network stability can be solved by multiple connections. The probability of multiple connections being disconnected at the same time is lower than the probability of a single connection being disconnected in a single host.

- A transmission mechanism is designed for multi-homed systems. The transmission mechanism can be changed in a variety of Wi-Fi network environments. In a wireless environment, the device can choose a variety of data transmission methods, which enhances the flexibility of the system.

- Combining the SUBN structure and the indirect similarity of SUBN reasoning, a method of indExperiments show that the system and protocol can enhance the stability of multimedia data transmission. In a multi-homed system, sensors can enhance the stability of the system under the same resource consumption conditions. Multi-homed sensors are more stable than single-homed sensors under the same CPU and memory consumption. 
- An efficient routing schema in multi-homing scenario is presented based on protocoloblivious forwarding (POF).In the proposed schema,the packet forwarded to the multi-homed host carries multiple destination addresses to obtain the ability of switching the transmission path;meanwhile,the router dynamically adjusts the path of the packet through the perception of the network status.

For Wireless Multimedia Sensor Networks (WMSN), how to increase the throughput in constrained environments has become the main challenge. Multipath routing is a promising solution because it can improve the channel utilization rate, reduce transmission delay and balance the transmission load. To pursue high efficiency for a multipath routing scheme, the characteristics of wireless environments and sensor networks are researched into careful considerations. In this paper, a new multi-homed connection mode is designed, which divides the whole network topology into many districts and simultaneously forwards data through these districts without interfering with each other - to achieve interference-free transmissions. The load of each district is will adjusted according to the energy status of their nodes, it does not establish fixed paths in advance and will hence maintain high performance even when the topology changes rapidly. When compared with related routing schemes, our scheme can achieve higher performance at real-time transmission and meanwhile distribute transmission loads more evenly to most of the nodes in the network.

\section{FUNDING AGENCY}

Publisher has waived the Open Access publishing fee.

\section{ACKNOWLEDGMENT}

This work was supported by the Scientific Research Project (No: 20B338) of Hunan Provincial Education Department, China. 


\section{REFERENCES}

Al-Najjar, A., Khan, F. H., \& Portmann, M. (2020). Network traffic control for multi-homed end-hosts via SDN. IET Communications, 14(19), 3312-3323. doi:10.1049/iet-com.2020.0335

Aljarajreh, H., Lu, D. C., Siwakoti, Y. P., Aguilera, R. P., \& Chi, K. T. (2021). A Method of Seamless Transitions Between Different Operating Modes for Three-Port DC-DC Converters. IEEE Access: Practical Innovations, Open Solutions, 9, 59184-59195. doi:10.1109/ACCESS.2021.3073948

Arena, F., \& Pau, G. (2020). An overview of big data analysis. Bulletin of Electrical Engineering and Informatics, 9(4), 1646-1653. doi:10.11591/eei.v9i4.2359

Capela, N., \& Sargento, S. (2014). Multihoming and network coding: A new approach to optimize the network performance. Computer Networks, 75, 18-36. doi:10.1016/j.comnet.2014.09.002

Chaari, H., Mnif, K., Zarai, F., \& Kamoun, L. (2020). New Bandwidth Upgradation and Degradation Algorithms for Next Generation of Wireless Network Systems. Wireless Personal Communications, 113(1), 79-97. doi:10.1007/s11277-020-07179-4

Chaudhary, D. D., \& Waghmare, L. M. (2013). Energy efficiency and latency improving protocol for wireless sensor networks. Proceedings of the 2013 International Conference on Advances in Computing, Communications and Informatics, 1303-1308. doi:10.1109/ICACCI.2013.6637366

Giliberto, M., Arena, F., \& Pau, G. (2019). A fuzzy-based Solution for Optimized Management of Energy Consumption in e-bikes. Journal of Wireless Mobile Networks, Ubiquitous Computing and Dependable Applications, 10(3), 45-64. doi:10.22667/JOWUA.2019.09.30.045

Joshi, Y. K., \& Younis, M. (2014). Straight skeleton based reconnection in a wireless sensor network. Proceedings of the 2014 Global Communications Conference, 283-288. doi:10.1109/GLOCOM.2014.7036821

Kim, D., \& Chung, K. (2014). Adaptive multiple TCP-connection scheme to improve video quality over wireless networks. Transactions on Internet and Information Systems (Seoul), 8(11), 4068-4086.

Kuntz, R., Montavont, J., \& Noel, T. (2013). Multihoming in IPv6 mobile networks: Progress, challenges, and solutions. IEEE Communications Magazine, 51(1), 128-135. doi:10.1109/MCOM.2013.6400449

Lee, H. C., Chang, Y. C., \& Huang, Y. S. (2014). A reliable wireless sensor system for monitoring mechanical wear-out of parts. IEEE Transactions on Instrumentation and Measurement, 63(10), 2488-2497. doi:10.1109/ TIM.2014.2312498

Li, G. H., \& Li, J. Z. (2011). Global weighted fairness guaranteed congestion avoidance protocol for wireless sensor networks. Proceedings of the 30th International Conference on Computer Communications, Joint Conference of the IEEE Computer and Communications Societies, 601-605. doi:10.1109/INFCOM.2011.5935235

Liu, Y. C., Jiantao Zhang, J. T., Chi, K. T., Zhu, C. B., \& Wong, S. C. (2021). General Pathways to Higher Order Compensation Circuits for IPT Converters via Sensitivity Analysis. IEEE Transactions on Power Electronics, 36(9), 9897-9906. doi:10.1109/TPEL.2021.3062228

Ma, P. F., You, J. L., \& Wang, J. L. (2020). An efficient multipath routing schema in multi-homing scenario based on protocol-oblivious forwarding. Frontiers of Computer Science, 14(4), 95-105. doi:10.1007/s11704-019-8397-5

Nguyen, X. T., Nguyen, H. T., \& Pham, C. K. (2015). SAR: a self-adaptive and reliable protocol for wireless multimedia sensor networks. Proceedings of the 7th International Conference on Ubiquitous and Future Networks, 760-765.

Park, J. W., Karrer, R. P., \& Kim, J. W. (2011). TCP-ROME: A transport-layer parallel streaming protocol for real-time online multimedia environments. Journal of Communications and Networks (Seoul), 13(3), $277-285$. doi:10.1109/JCN.2011.6157437

Radhika, M., \& Sivakumar, P. (2021). Energy optimized micro genetic algorithm based LEACH protocol for WSN. Wireless Networks, 27(1), 27-40. doi:10.1007/s11276-020-02435-8

Sarisaray-Boluk, P., \& Akkaya, K. (2015). Performance comparison of data reduction techniques for wireless multimedia sensor network applications. International Journal of Distributed Sensor Networks, 2015(8), 1-15. doi: $10.1155 / 2015 / 873495$ 
Souihi, S., Souidi, M., \& Mellouk, A. (2016). An adaptive QoE-based network interface selection for multi- homed eHealth devices. Proceedings of the 2nd International Internet of Things Summit, 437-442. doi:10.1007/9783-319-47063-4_45

Sousa, B. M., Pentikousis, K., \& Curado, M. (2011). Multihoming management for future networks. Mobile Networks and Applications, 16(4), 505-517. doi:10.1007/s11036-011-0323-5

Wallace, T. D., \& Shami, A. (2012). A review of multihoming issues using the stream control transmission protocol. IEEE Communications Surveys and Tutorials, 14(2), 565-578. doi:10.1109/SURV.2011.051111.00096

Wang, J. Y., Wen, J. T., \& Zhang, J. (2011). TCP- FIT: an improved TCP congestion control algorithm and its performance. Proceedings of the 30th International Conference on Computer Communications, Joint Conference of the IEEE Computer and Communications Societies, 2894-2902. doi:10.1109/INFCOM.2011.5935128

Wu, J. Y., Shang, Y. L., Cheng, B., Wu, B., \& Chen, J. (2014). Loss tolerant bandwidth aggregation for multihomed video streaming over heterogeneous wireless networks. Wireless Personal Communications, 75(2), 1265-1282. doi:10.1007/s11277-013-1422-3

Wu, J. Y., Yuen, C., Cheung, N. M., \& Chen, J. (2016). Delay- constrained high definition video transmission in heterogeneous wireless networks with multi- homed terminals. IEEE Transactions on Mobile Computing, 15(3), 641-655. doi:10.1109/TMC.2015.2426710

Zam, A., Khayyambashi, M. R., \& Bohlooli, A. (2020). Energy-efficient face detection and recognition scheme for wireless visual sensor networks. Applied Soft Computing, 89(6), 106014. doi:10.1016/j.asoc.2019.106014

Haitao Wang (b. 1982) received the B.S. degree in Information and Computing Sciences from Changsha University of Science \& Technology in 2004, and received the M.S. degree in Technology of Computer Application from Hunan University in 2011. Now, he is a lecturer at College of Computer, Hunan International Economics University, China. His research interests include network and information security.

Yanli Chen (b. 1982) received the B.S. degree in Computer Science and Technology from Hunan Normal University in 2005 and received the M.S. degree in Computer Science and Technology from Hunan University in 2010. Now, she is a lecturer at College of Computer, Hunan University of International Economics, China. Her research interests include image processing and information security. 